\title{
The Effect Chemical Reaction to Free Convection of Micropolar Fluid in a Vertical Channel
}

\author{
Yulita Molliq Rangkuti ${ }^{1}$, Hermawan Syahputra ${ }^{2}$ and Habibis Saleh $^{3}$ \\ \{yulitamolliq@ unimed.ac.id ${ }^{1}$, hsyahputra@unimed.ac.id ${ }^{1}$,dr.habibissaleh@gmail.com ${ }^{3}$ \} \\ Department of Mathematics, State University of Medan, Medan 20221, North Sumatera, Indonesia ${ }^{1}$, \\ Department of Mathematics, University of Riau, Pekanbaru,28293, Riau, Indonesia ${ }^{2,3}$
}

\begin{abstract}
The present analysis is focused to the criteria for the onset of flow inversion of the fully developed free convection of micropolar fluid in a vertical channel under the effect of the chemical reaction. The governing equations force are solved and calculated numerically by Shooting Method based on Fourth order Runge Kutta Method. Parameter for the occurrence of invented flow by was presented. The exothermic chemical reaction is adopted to gain the optimally velocity, microrotasi and temperature.
\end{abstract}

Keywords: Micropolar Fluid; Chemical Reaction; Free Convection

\section{Introduction}

Micropolar fluids suggested by [1]simulated accurately the flow characteristics of polymeric additives, geomorphological sediments, colloidal and haematological suspensions, liquid crystals, lubricants etc. Studies of the flows of heat convection in micropolar fluids focus mainly on flat surfaces by Rahman et al. in[7]-[11]. [13] have considered natural convection flow of micropolar fluid along a vertical and a permeable semi-infinite plate embedded in a porous medium. The problem of fully developed natural convection heat and mass transfer of a micropolar fluid between porous vertical plates with asymmetric wall temperatures and concentrations is analyzed by [14].

Flow of fluids with internal heat sources are of great practical and theoretical interest. The fluid motion develops slowly following the development of non-uniformity in the temperature field. The volumetric heat generation/ absorption term exerts strong effectto the flow when the temperature difference is appreciably large. The analysis of temperature field as modified by the heat source in a moving fluid is important in view of chemical reactions. One of the earlieststudieson free convection of micropolar fluid inaverticalchannelwith uniform wall temperatures had been studied analytically by [2].

Foraboschi and Federico in [5] have assumed 2 state volumetric heat generation as depending on temperature difference

$$
\theta= \begin{cases}\theta_{0}\left(\mathrm{~T}-\mathrm{T}_{0}\right), \mathrm{T} \geq \mathrm{T}_{0} \\ 0, \mathrm{~T}>\mathrm{T}_{0}\end{cases}
$$

In many chemical engineering processes chemical reactions take place between a foreign mass and the working fluid which moves due to stretching or otherwise of a surface. A chemical reaction is said to be first order and homogenous if its rate of reaction is directly proportional to the concentration and it occurs as a single phase volume reaction. Muthucumaraswamy studied the effects of a chemical reaction on a moving isothermal 
vertical surface with suction and [3]considered MHD free convection flow and mass transfer over a stretching sheet with chemical reaction [6].

In this paper, an model is bulit with effect of chemical reaction by varous parameters to micropolar fluid

\section{Mathematical Formulation}

Consider a laminar free convection flow from the micropolar fluid between two solid and thick plates between two parallel and vertical walls. The space between the plates is $\mathrm{h}$. Walls at $\mathrm{y}=0$ and $\mathrm{y}=\mathrm{h}$ are isothermal at certain temperatures $\mathrm{T}_{1}$ and $\mathrm{T}_{2}$, where it is assumed that $\mathrm{T}_{1} \geq \mathrm{T}_{2}$. The fluid has a vertical velocity distribution evenly up the $\mathrm{U}_{0}$ stream at the entrance of the channel. Thus, the basic equation for asteady and fully developed flow from a thick, non-compactable and heat fluid that is assumed to be supplied to the surrounding fluid in[4] by an exothermic surface reaction [12]. On the basis of this assumption, the equation describes the physical situation

Subject to boundary condition

$$
\begin{aligned}
& (\mu+\kappa) \frac{d^{2} u}{d y^{2}}+\kappa \frac{d n}{d y}+\rho g \beta\left(T-T_{0}\right)=0 \\
& \gamma \frac{d^{2} n}{d y^{2}}-\kappa\left(2 n+\frac{d u}{d y}\right)=0 \\
& \frac{d^{2} T}{d y^{2}}=Q k_{0} a e^{-\frac{E}{R T}}
\end{aligned}
$$

$$
\begin{array}{lll}
\mathrm{u}(0)=0, & \mathrm{~T}(0)=\mathrm{T}_{1}, & \mathrm{n}(0)=0 \\
\mathrm{u}(\mathrm{h})=0 & \mathrm{~T}(\mathrm{~h})=\mathrm{T}_{2} & \mathrm{n}(\mathrm{h})=0
\end{array}
$$

Reactions occur only on surfaces that are constructed by Arrhenius first order kinetic. The closure system by conservation of mass flux $M$ is $\int \mathrm{U} \mathrm{dY}=\mathrm{M}$ [4]. The construction of nondimensional equations for this equation becomes

$$
\begin{aligned}
& (1+K) \frac{d^{2} U}{d Y^{2}}+K \frac{d N}{d Y}+\theta=0 \\
& \left(1+\frac{K}{2}\right) \frac{d^{2} N}{d Y^{2}}-K\left(2 N+\frac{d U}{d Y}\right)=0 \\
& \frac{d^{2} \theta}{d Y^{2}}=-K_{F} e^{\theta}
\end{aligned}
$$

Where $j=h^{2}$ and $K$ is material parameter which defined by

$$
\begin{gathered}
\gamma=\left(1+\frac{\kappa}{2 \mu}\right) h^{2}=\left(1+\frac{K}{2}\right) h^{2} \\
K=\frac{k}{\mu}
\end{gathered}
$$

boundary condition be

$$
\begin{array}{ccc}
\mathrm{U}(0)=0, & \theta(0)=\mathrm{R}_{\mathrm{T}}, & \mathrm{N}(0)=0 \\
\mathrm{U}(1)=0 & \theta(1)=-\mathrm{R}_{\mathrm{T}}, & \mathrm{N}(1)=0
\end{array}
$$

Here, effect of micropolar based on $K, N \neq 0$.

\section{Result And Discussion}

The effects of chemical reaction by various $K, K_{F}$, and $R T$ are presented in Figure 1-3, respectively. Figure 1: First figures show that velocity increases as value $K$, maximum velocity was obtained for $K$ value was getting big. Whereas, velocity increase as $R_{T}$ small. I 
velocity is maximum when Frank Kamenetskii number $K_{F}=1.5$. Figure 2:Figures 2 show that microrotation decreases as various value of material parameter $K$.Microrotation is maximum when temperature different ratio $\left(R_{T}\right)$ is getting big. Whereas, microrotation has minimum value for various value of $K_{F}$ for $\mathrm{Y}<0.5$, while, microrotation has maximum value for various value of $K_{F}$ for $\mathrm{Y} \geq 0.5$. Finally, microrotation approach optimal when $K_{F}=1.5$. Figure 3: Figures 3 show that temperature has maximum value as various value of material parameter $\mathrm{K}$ at $\mathrm{Y}=0,4$, Temperature is maximum at $\mathrm{Y} \geq 0,45$ for temperatur different ratio $\left(R_{T}=0.1\right)$ and Temperature is maximum at $\mathrm{Y}<0,45$ for temperature different ratio $\left(R_{T}=1\right)$. Finally, temperature approach maximum when $K_{F}=1$.

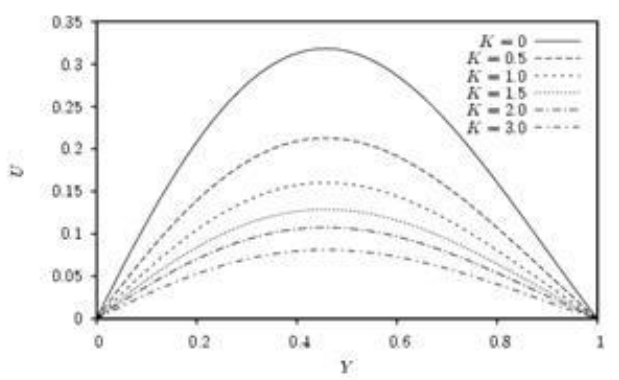

(a)

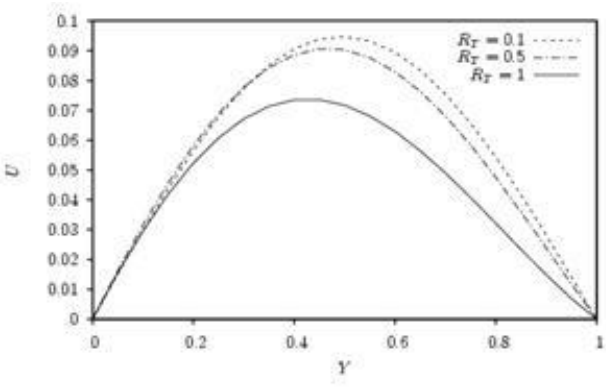

(b)

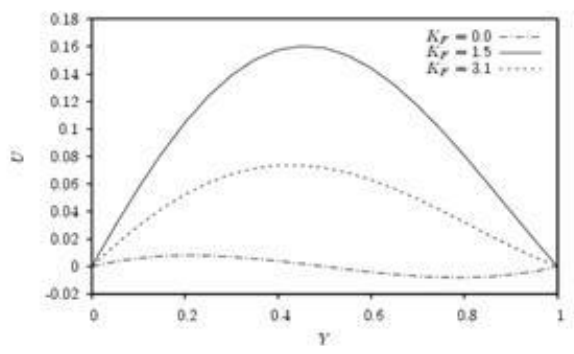

(c)

Fig. 1. Plots of $U$ versus $Y$ for different values of (a) $\mathrm{K}$ where $\mathrm{K}_{\mathrm{F}}=1.5, \mathrm{R}_{\mathrm{T}}=1.0,(\mathrm{~b}) \mathrm{K}_{\mathrm{F}}$ where $\mathrm{R}_{\mathrm{T}}=1.0$, $\mathrm{K}=1.0$ and (c) $\mathrm{R}_{\mathrm{T}}$ where $\mathrm{K}_{\mathrm{F}}=1.5, \mathrm{~K}=1.0$ 


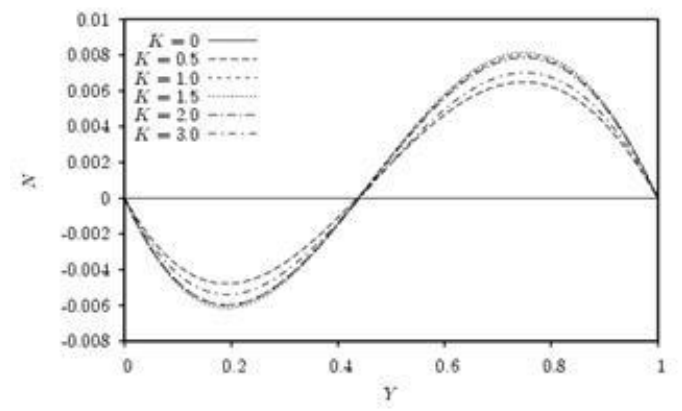

(a)

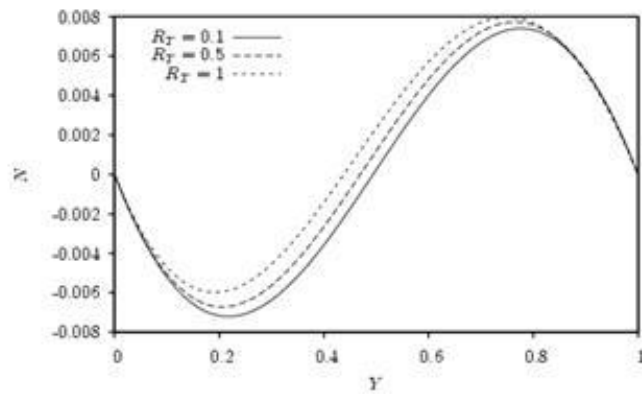

(b)

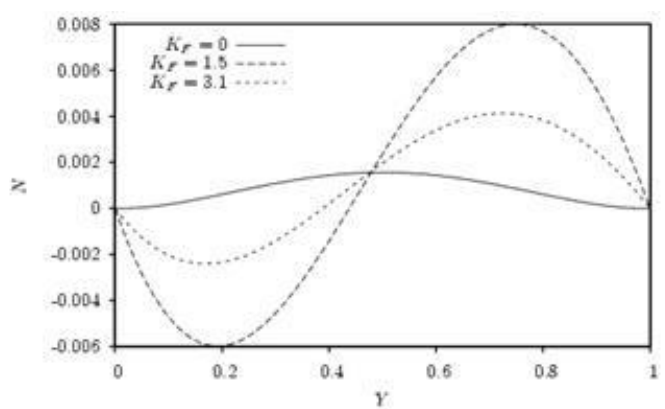

(c)

Fig. 2. Plots of $\mathrm{N}$ versus $Y$ for different values of (a) $\mathrm{K}$ where $\mathrm{K}_{\mathrm{F}}=1.5, \mathrm{R}_{\mathrm{T}}=1.0,(\mathrm{~b}) \mathrm{K}_{\mathrm{F}}$ where $\mathrm{R}_{\mathrm{T}}=1.0$, $\mathrm{K}=1.0$ and (c) $\mathrm{R}_{\mathrm{T}}$ where $\mathrm{K}_{\mathrm{F}}=1.5, \mathrm{~K}=1.0$ 


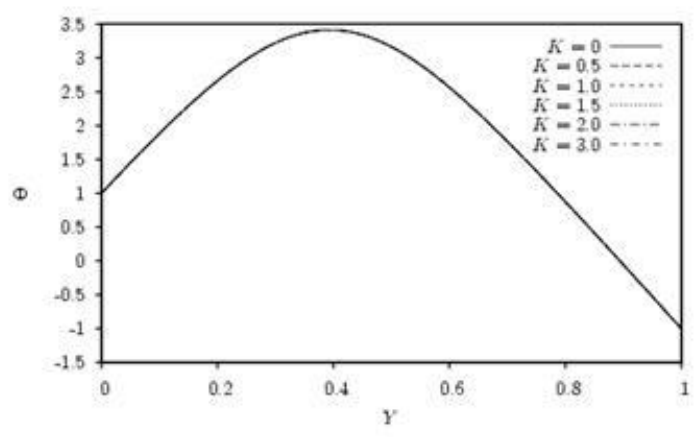

(a)

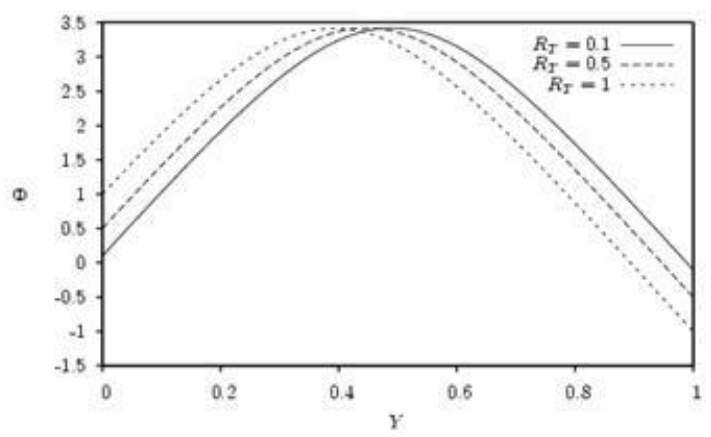

(b)

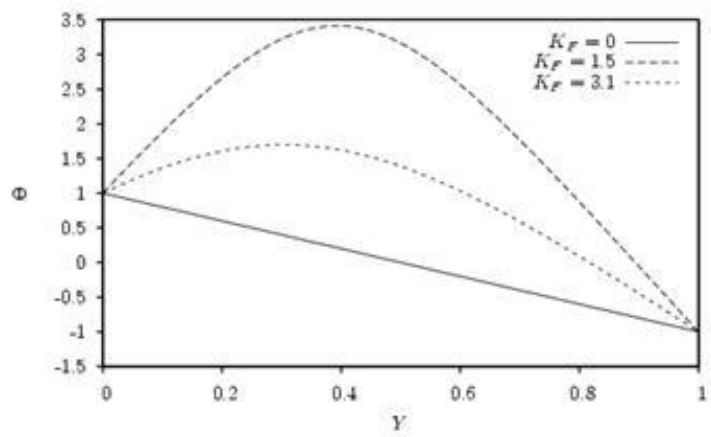

(c)

Fig. 3. Plots of $\Theta$ versus $Y$ for different values of (a) $\mathrm{K}$ where $\mathrm{K}_{\mathrm{F}}=1.5, \mathrm{R}_{\mathrm{T}}=1.0,(\mathrm{~b}) \mathrm{K}_{\mathrm{F}}$ where $\mathrm{R}_{\mathrm{T}}=1.0$, $\mathrm{K}=1.0$ and (c) $\mathrm{R}_{\mathrm{T}}$ where $\mathrm{K}_{\mathrm{F}}=1.5, \mathrm{~K}=1.0$

\section{Conclusions}

Parameters for the occurrences flow reversal by freeconvection of Mocrpolar fluid under the effect of chemical reaction in a vertical channel are presented. It can be concluded that flow reversal adjacent to the cold wall is found to exist with in the channel as the ratio of Frank-Kamenetskiinumber and Reynolds number is above a thre shold value. The exothermic 
chemical reaction is found to enchance the flow reversal and made flow reversal possible for symmetrical walls temperatures.

\section{Aknowledgement}

The financial support received from BOPTN UNIMED is gratefully acknowledged.

\section{Nomenclature}

$u \quad$ non-dimensional velocity, $\mathrm{m} / \mathrm{s}$.

$T$ non-dimensional temperature

$T_{0} \quad$ reference temperature

$t \quad$ non-dimensional time, $\mathrm{s}$

$K_{F} \quad$ Frank-Kamenetskiinumber

$g \quad$ acceleration due to gravity

$R_{T} \quad$ Temperaturedifferenceratio

$k \quad$ kinematic rotational viscosity, N.s $/ \mathrm{m}^{2}$

$n \quad$ Micro-rotation velocity

$\rho \quad$ Density of fluid

$U \quad$ Dimensionlessvelocitycomponentsin the $x$-direction

$X, Y \quad$ Dimensionlessspacecoordinates

$\Theta \quad$ Dimensionlesstemperature

$K \quad$ Non-dimensional material Parameter

\section{References}

[1] A.C.Eringen.: Theory of micropolar fluids. Journal of Mathematics and Mechanics. Vol. 16, pp. 1-18. (1966)

[2] W. Aung.:Fully Developed Laminar Free Convection Between Vertical Plates Heated Asymmetrically, International Journal of Heat Mass Transfer. Vol.15. pp. 1577-1580.(1972)

[3] A.A.Afify, MHD.: free convective flow and mass transfer over a stretching sheet with chemical reaction. Heat Mass Transfer. Vol. 40, pp. 495-500. (2004)

[4] A.J.Chamka, T.Grosan, \&I. Pop.: fully developed free convection of micropolar fluid in a vertical channel International Communications in Heat and Mass Transfer. Vol. 29, pp. 1119-1127. (2002)

[5] A.C.Eringen.: Theory of micropolar fluids. Journal of Mathematics and Mechanics. Vol. 16, pp. 1-18. (1966)

[6] F.P. Foraboschi, and I.D. Federico.: Heat transfer in laminar flow of non-Newtonian heatgenerating fluids. International Journal Heat Mass Transfer. Vol. 7, pp. 315-318.(1964)

[7] R. Muthucumaraswamy.: Effects of a chemical reaction on a moving isothermal vertical surface with suction. Acta Mechanics. Vol. 155, pp. 65-70. (2002)

[8] M.M. Rahman, I.A. Eltayeb, I.A.\& Rahman, S.M.M.: 2009a Thermo-micropolar fluid flow along a vertical permeable plate with uniform surface heat flux in the presence of heat generation. Thermal Science. Vol. 13, pp. 23-36. (2009)

[9] M.M.,Rahman.: Convective flows of micropolar fluids from radiative isothermal porous surfaces with viscous dissipation and joule heating. Communications in Nonlinear Scienceand Numerical Simulation. Vol. 14. (2009) 
[10] M.M. Rahman, and T. Sultana.: Radiative heat transfer flow of micropolar fluid with variable heat flux in a porous medium. Nonlinear Analysis-Modelling and Control. Vol. 13, pp. 71-87. (2008)

[11] M.M Rahman and T.Sultana.: Transient convective flow of micropolar fluid past a continuously moving vertical porous plate in the presence of radiation. International Journal of Applied Mechanics and Engineering. Vol. 12. (2007)

[12] M.M. Rahmanand M.A. Sattar.: MHD convective flow of a micropolar fluid past a continuously moving vertical porous plate in the presence of heat generation/absorption. ASME Journal Heat Transfer. Vol. 128, pp. 142-52. (2006)

[13] H. Saleh, I. Hashim. \&S. Asriati,.: Flow Reversal of Fully Developed Mixed Convection in a Vertical Channel with Chemical Reaction, International Journal of Chemical Engineering. Vol. 2013, Article ID 310273. (2010)

[14] I.A. Hassanien, A.H. Essawy\&N.M.Moursy.: MoursyNatural convection flow of micropolar fluid from a permeable uniform heat flux surface in porous medium. Applied Mathatematics Computtion. Vol. 152.

[15] O.Abdulazizand I.Hashim.: Fully developed free convection heat and mass transfer of a micropolar fluid between porous vertical plates. Numerical Heat Transfer Part A: Applications. Vol. 55, pp. 270-88. (2009)

[1] A.C.Eringen.: Theory of micropolar fluids. Journal of Mathematics and Mechanics. Vol. 16, pp. 1-18. (1966)

[12]Somov, A.: Wildfire safety with wireless sensor networks. EAI Endorsed Transactions on Ambient Systems. pp. 1-11 (2011)

[13]Motaz, A.: Start programming using Object Pascal. Vol. 2, pp. 10-11. Legally Free Computer Books, US (2013) 